\title{
Evaluation of Circulating Endothelial Progenitor Cells in Abdominal Aortic Aneurysms after Endovascular Aneurysm Repair
}

\author{
Weihua $\mathrm{Wu}^{1,2, *}$, Jinlong Zhang ${ }^{1, *}$, Lianbo Shao, ${ }^{1, *}$ \\ Haoyue Huang ${ }^{1}$, Qingyou Meng ${ }^{1}$, Zhenya Shen ${ }^{1}$, Xiaomei Teng \\ ${ }^{I}$ Department of Cardiovascular Surgery of the First Affiliated Hospital $\mathcal{E}$ Institute for Cardiovascular Science, Soochow University, \\ Suzhou, China \\ ${ }^{2}$ Center of Clinical Laboratory, The First Affiliated Hospital of Soochow University, Suzhou, China
}

Background and Objectives: Circulating endothelial progenitor cells (EPCs) participate in vascular repair and predict cardiovascular outcomes. The aim of this study was to investigate the correlation between EPCs and abdominal aortic aneurysms (AAAs).

Methods and Results: Patients (age 67 \pm 9.41 years) suffering from AAAs (aortic diameters $58.09 \pm 11.24 \mathrm{~mm}$ ) were prospectively enrolled in this study. All patients received endovascular aneurysm repair (EVAR). Blood samples were taken preoperatively and 14 days after surgery from patients with aortic aneurysms. Samples were also obtained from age-matched control subjects. Circulating EPCs were defined as those cells that were double positive for CD34 and CD309. Rat models of AAA formation were generated by the peri-adventitial elastase application of either saline solution (control; $n=10$ ), or porcine pancreatic elastase (PPE; $n=14)$. The aortas were analyzed using an ultrasonic video system and immunohistochemistry. The levels of $\mathrm{CD} 34^{+} / \mathrm{CD} 309^{+}$cells in the peripheral blood mononuclear cell populations were measured by flow cytometry. The baseline numbers of circulating EPCs $\left(\mathrm{CD} 34^{+} / \mathrm{CD} 309^{+}\right)$in the peripheral blood were significantly smaller in AAA patients compared with control subjects. The number of EPCs doubled by the 14th day after EVAR. A total of $78.57 \%$ of rats in the PPE group (11/14) formed AAAs (dilation ratio $>150 \%$ ). The numbers of EPCs from defined AAA rats were significantly decreased compared with the control group. Conclusions: EPC levels may be useful for monitoring abdominal aorta aneurysms and rise after EVAR in patients with aortic aneurysms, and might contribute to the rapid endothelialization of vessels.

Keywords: Abdominal aortic aneurysms, Endothelial progenitor cells, Endovascular aneurysm repair, Porcine pancreatic elastase

Received: February 3, 2021, Revised: June 11, 2021, Accepted: August 2, 2021, Published online: October 31, 2021

Correspondence to Xiaomei Teng

Department of Cardiovascular Surgery of the First Affiliated Hospital \& Institute for Cardiovascular Science, Soochow University, 899 Pinghai Road, Suzhou 215000, China

Tel: +86-051267780946, Fax: +86-051267780946, E-mail: tengxiaomei@suda.edu.cn

Co-Correspondence to Zhenya Shen

Department of Cardiovascular Surgery of the First Affiliated Hospital \& Institute for Cardiovascular Science, Soochow University, 899 Pinghai Road, Suzhou 215000, China

Tel: +86-051267972066, Fax: +86-051267972066, E-mail: uuzyshen@aliyun.com

Co-Correspondence to Qingyou Meng

Department of Cardiovascular Surgery of the First Affiliated Hospital \& Institute for Cardiovascular Science, Soochow University, 899 Pinghai Road, Suzhou 215000, China

Tel: +86-051267780948, Fax: +86-051267780948, E-mail: mengqy@163.com

${ }^{*}$ These authors contributed equally to this work.

다 This is an open-access article distributed under the terms of the Creative Commons Attribution Non-Commercial License (http://creativecommons.org/licenses/by-nc/4.0/), which permits unrestricted non-commercial use, distribution, and reproduction in any medium, provided the original work is properly cited.

Copyright (c) 2022 by the Korean Society for Stem Cell Research 


\section{Introduction}

Abdominal aortic aneurysms (AAAs) are an abnormal focal dilation of aortic vessels in the abdominal area. AAAs are defined as having a diameter for a dilated aorta measuring $50 \%$ greater than the proximal normal segment, or $>3 \mathrm{~cm}$ in maximum. The majority of AAAs are asymptomatic and are most often incidentally found upon physical examination (1). In aging populations, the incidence and prevalence of AAAs are certain to rise (2).

Diseases and unhealthy behaviors that damage the heart and blood vessels also increase risks for aortic aneurysm, such as smoking, hypertension, hypercholesterolemia, and atherosclerosis (3). Numerous studies have suggested that endothelial progenitor cells (EPCs) can be used as a biomarker of cardiovascular diseases $(4,5)$. Studies have shown different results with regards to the correlation between the number of EPCs and aortic aneurysm prevalence. In a study by Dawson, AAA patients had significantly higher levels of circulating EPCs $\left(\mathrm{CD} 34^{+} / \mathrm{CD} 133^{+}\right)$than age-matched controls (6). However, circulating EPCs $\left(\mathrm{CD} 34^{+} / \mathrm{KDR}^{+}\right.$and $\left.\mathrm{CD} 133^{+} / \mathrm{KDR}^{+}\right)$were significantly fewer in AAA patients than in controls in Chen's study (7). The significant controversies in these diametrically opposed results could be related to the identification methods and roles of EPC subpopulations and patient groups.

EPCs are a heterogeneous group of cells, which are present in circulatory populations and contribute to vasculogenesis in adults (8). EPCs can be isolated from hematopoietic sources or nonhematopoietic tissues. Circulating EPCs have been characterized by coexpression of surface markers such as CD34, CD133, and CD309 (also known as VEGFR-2 or KDR). Previous studies have investigated the inconsistent number of EPCs in AAA patients compared with matched controls.

Endovascular aneurysm repair (EVAR) was first reported by Parodi in 1991 and has gained in popularity as a minimally invasive procedure to repair abdominal aortic aneurysm (9-11). It remains unclear whether changes in circulating EPCs occur in AAA patients after EVAR. In order to address this, we investigated the number of circulating EPCs in patients with AAAs before or after EVAR. We further uncovered a quantitative relationship in the number of EPCs using a rodent model of aortic aneurysms.

\section{Materials and Methods}

\section{Study population}

Patients referred for Computed Tomography Angiography
(CTA) with suspected AAA were recruited from June 2018 to October 2018. Inclusion criteria for the study were men and women aged 50 85 years with a dilation aorta diameter over $50 \%$ that of a normal aorta. Appropriate exclusion criteria were aortic aneurysms secondary to genetic syndromes such as Marfan syndrome, unstable angina, recent trauma, underlying infection, and cancer. All patients suffering from unruptured AAAs underwent endovascular aneurysm repair (EVAR) and provided written informed consent the day before surgery. A control group had a normal abdominal aorta diameter according to the principle of matched grouping. Healthy volunteers were recruited from Soochow University post-graduate students and other young subjects with a mean age of 26 years.

The protocol for this study was approved by the Institutional Review Board of the first affiliated hospital of Soochow University, and informed consent was obtained from all enrolled patients.

\section{Collection of peripheral blood samples}

Peripheral blood $(5 \mathrm{ml})$ was collected in EDTA-coated tubes from patients with AAAs who underwent EVAR $(n=7)$. Samples were taken preoperatively and on the 14th day after surgery. Blood samples were also obtained from matched control subjects $(n=7)$ and other young subjects $(\mathrm{n}=6)$. Peripheral blood mononuclear cells (PBMNs) were isolated using density gradient centrifugation with Ficoll separating solution (Beyotime, Shanghai, China). The serum levels of creatinine, total cholesterol, and triglycerides were determined at admission and on the 14th day after surgery.

\section{Quantification of circulating EPCs by flow cytometry}

The numbers of EPCs in each sample were assessed according to a previously published technique $(12,13)$. Circulating EPCs were measured using flow cytometry and quantified as PBMNs co-expressing CD34 (a hematopoietic stem and EPC marker) and CD309 (a vascular endothelial growth factor receptor). In brief, we incubated $200 \mu 1$ of PBMNs with $5 \mu 1$ of FITC-conjugated antiCD34 antibody (eBioscience, San Diego, USA) and PEconjugated anti-CD309 antibody (eBioscience, San Diego, USA) in the dark for $15 \mathrm{~min}$. After incubation, each sample was washed twice with FACS buffer. Approximately $10^{4}$ events per sample were run on a Guava ${ }^{\mathbb{R}}$ easyCyte 8 Flow Cytometer (Millipore Corporation, Billerica, USA), and samples were analyzed with FlowJo software (Tree Star, Ashland, USA). 


\section{Animal models}

A rat model of AAA formation was generated using peri-adventitial elastase application (14). Briefly, 8-week-old male Sprague-Dawley rats (Animal Laboratory of Soochow University, China) were assigned randomly to two groups, a control group $(\mathrm{n}=10)$ and a porcine pancreatic elastase (PPE) group $(\mathrm{n}=14)$. For experiments, rats were anesthetized by an intra-peritoneal injection of $10 \%$ chloral hydrate $(350 \mathrm{mg} / \mathrm{kg})$. A laparotomy was performed, and the abdominal aorta was exposed just below the left renal vein. These isolated segments of the abdominal aorta were perivascularly treated for 15 minutes with $7 \mathrm{U} / \mathrm{mg}$ PPE (Type I; Sigma, St. Louis, USA) or a saline solution using a presoaked gauze. After the removal of residual elastase with saline solution, the wounds were closed, and all rats were returned to their cages and fed with standard diet chow and water. All animal care and use in these experiments conformed to the Guidelines of the Animal Experimental Committee of Soochow University.

\section{Measurements of the abdominal aorta diameter}

The survival rate was measured after elastase treatment for 5 weeks. Aneurysmal morphology and diameter were examined in each group using a high resolution small animal ultrasonic real-time video system (VisualSonics Vevo770, Canada). The entire aorta was harvested at the end of the 5th week, and then analyzed using histology. The dilation ratio $(\%)=($ Aneurysmal diameter/aortic diameter $) \times 100 \%$. AAA was defined as having a dilation ratio of more than $150 \%$.

\section{Histological and immunofluorescent analyses}

Aortic tissues from mice after 5 weeks of PPE or saline solution application were embedded in 4\% paraformaldehyde, and then cut into $5 \mu \mathrm{m}$ sections. The sections were stained with hematoxylin and eosin (H\&E) using a standard protocol, or Verhoeff-Van Gieson (VVG) staining for elastic fibers. The aortic sections were stained with Verhoeff's solution, ferric chloride, and Van Gieson's solution. After each staining cycle, a fixing and washing procedure followed. Aortic sections were incubated with primary antibodies overnight at $4^{\circ} \mathrm{C}$ using either a rabbit CD34 polyclonal (Abcam, Cambridge, MA, USA) or a rabbit CD309 (VEGF receptor 2) polyclonal antibody (Abcam, Cambridge, MA, USA), followed by incubation with secondary antibody conjugated with FITC (green) or TRITC (red). DAPI (blue) was used to counterstain the nucleus. Double staining was performed, and combinations of secondary antibodies with conjugated FITC and TRITC fluoresced as orange. Slides were imaged using an
Olympus BX61 Microscope (Olympus Corp, Japan).

\section{Statistical analyses}

Data is expressed as the mean \pm SD. Data was analyzed using an unpaired t-test between two groups, whereas one-way ANOVA was applied to compare more than two groups. A p-value of $<0.05$ was considered significant. All statistical analyses were performed using GraphPad Prism 8.0 software.

\section{Results}

\section{Study population}

All operations were scheduled and consisted of placement of a stent-graft (diameter 22 or $24 \mathrm{~mm}$ ) in each aneurysm. The study population was predominantly male $(71 \%)$. The mean age was $67 \pm 9.41$ years. Six patients $(86 \%)$ had hypertension and utilized one or more medications as anti-hypertensive therapy to control a systolic pressure of nearly $130 \mathrm{mmHg}$. Control and AAA patient's features before or after EVAR are summarized in Table 1. Most characteristic distributions between these groups were similar, but patients with AAAs had a larger median aneurysmal diameter and a lower total cholesterol level.

\section{Circulating number of EPCs populations in AAA patients}

The numbers of EPCs in the peripheral blood were measured by flow cytometry based on the percentage of CD $34^{+}$cells that co-expressed CD309 in patients and control subjects. The levels of circulating $\mathrm{CD} 34^{+} / \mathrm{CD} 309^{+}$ cells are shown in Fig. $1 . \mathrm{CD} 34^{+} / \mathrm{CD} 309^{+}$cell counts showed a significant decrease in patients with AAAs compared to control subjects. The levels of $\mathrm{CD} 34^{+} / \mathrm{CD} 309^{+}$ cells returned to pre aneurysm levels by the 14th day after EVAR.

\section{Peri-adventitial elastase creates aneurysms}

To demonstrate the relationship between EPCs and aneurysm, AAA rat models were established using peri-adventitial elastase application. No technical failures occurred intraoperatively. During the postoperative 5 weeks, there were no deaths in the control or PPE groups. The total survival rate was $100 \%$, and no significant difference was found between these groups. The mean max diameters of the abdominal aortas were $1.60 \pm 0.11 \mathrm{~mm}$ among the 24 rats as a baseline. At the end of the 5 weeks after operation, there were no differences in the aortic diameter in control animals, whereas the PPE group exposed to porcine pancreatic elastase displayed aortic dilation (Fig. 2A). 
Table 1. Subject's characteristics

\begin{tabular}{|c|c|c|c|c|}
\hline & Con $(n=7)$ & AAA before $\operatorname{EVAR}(n=7)$ & AAA after $\operatorname{EVAR}(n=7)$ & $\mathrm{p}$ \\
\hline Age, years & $69 \pm 9.81$ & $67 \pm 9.41$ & $67 \pm 9.41$ & N.S \\
\hline Male sex, $N(\%)$ & $4(57 \%)$ & $5(71 \%)$ & $5(71 \%)$ & N.S \\
\hline $\mathrm{SBP}, \mathrm{mmHg}$ & $132.14 \pm 10.62$ & $130.57 \pm 10.09$ & $130.14 \pm 6.33$ & N.S \\
\hline $\mathrm{DBP}, \mathrm{mmHg}$ & $73.57 \pm 6.26$ & $70 \pm 6.95$ & $71 \pm 10.18$ & N.S \\
\hline Hypertension (\%) & $5(71 \%)$ & $6(86 \%)$ & $6(86 \%)$ & N.S \\
\hline Diabetes (\%) & $1(14 \%)$ & $1(14 \%)$ & $1(14 \%)$ & N.S \\
\hline Smoking (\%) & $4(57 \%)$ & $4(57 \%)$ & $4(57 \%)$ & N.S \\
\hline CAD $(\%)$ & $2(29 \%)$ & $1(14 \%)$ & $1(14 \%)$ & N.S \\
\hline Diameter (mm) & $18.28 \pm 2.56$ & $58.09 \pm 11.24 *$ & $23.14 \pm 1.07^{\#}$ & $<0.05$ \\
\hline WBC $\left(10^{9} / \mathrm{l}\right)$ & $7.10 \pm 1.10$ & $6.68 \pm 1.41$ & $6.52 \pm 1.36$ & N.S \\
\hline Platelet $\left(10^{9} / \mathrm{l}\right)$ & $205.71 \pm 48.33$ & $214 \pm 49.06$ & $257.86 \pm 89.75$ & N.S \\
\hline Creatinine, $\mu \mathrm{mol} / \mathrm{l}$ & $71.56 \pm 21.93$ & $73.5 \pm 13.32$ & $71.69 \pm 7.73$ & N.S \\
\hline Hemoglobin, mmol/l & $31.01 \pm 1.15$ & $30.03 \pm 1.85$ & $29.24 \pm 1.97^{\#}$ & $<0.05$ \\
\hline Total cholesterol, $\mathrm{mmol} / \mathrm{l}$ & $4.89 \pm 1.26$ & $4.43 \pm 0.65^{*}$ & $3.93 \pm 0.99 *$ & $<0.05$ \\
\hline Triglyceride, $\mathrm{mmol} / / \mathrm{l}$ & $2.18 \pm 0.93$ & $1.23 \pm 0.28$ & $1.1 \pm 0.36$ & N.S \\
\hline
\end{tabular}

Demographic and clinical characteristics, comorbodity conditions, complications of all subjects. Data are displayed as means \pm SD or number (\%). AAA: abdominal aortic aneurysm, EVAR: endovascular aneurysm repair, DBP: diastolic blood pressure, SBP: systolic blood pressure, CAD: coronary artery disease, WBC: white blood cell. ${ }^{*} p<0.05$ compared to Con. $\# p<0.05$ compared to AAA before EVAR.

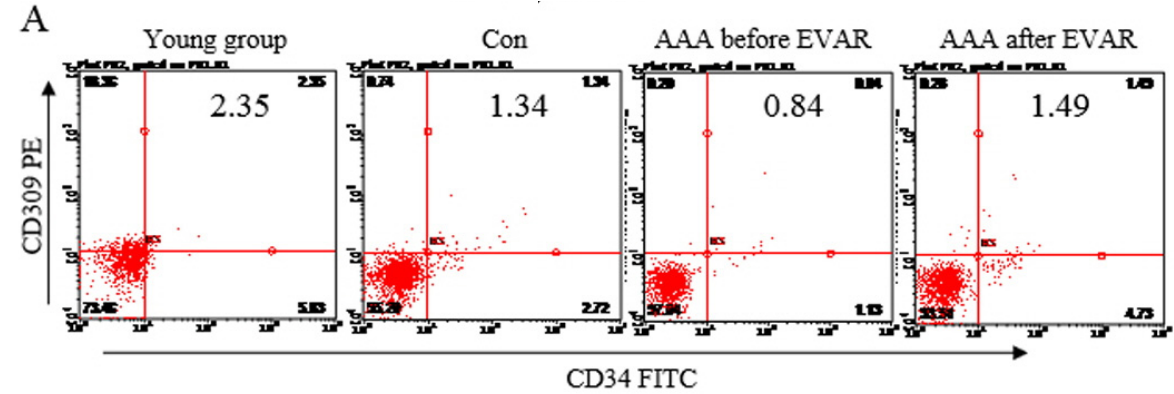

B

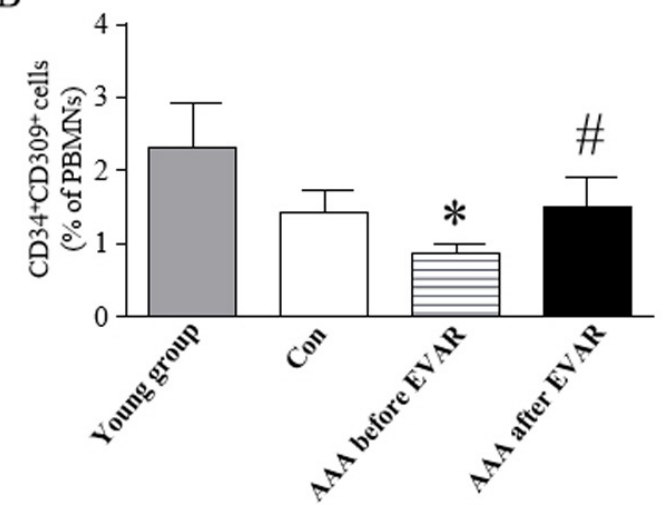

Fig. 1. Circulating levels of EPCs in the peripheral blood from subjects. (A) Circulating numbers of EPCs waswere measured in different groups by flow cytometry. (B) Percentage of circulating $\mathrm{CD}_{34}{ }^{+} / \mathrm{CD}_{30} 9^{+}$cells in patients with abdominal aortic aneurysms compared to age-matched controls. ${ }^{*} p<0.05$, vs. Con. $\# p<0.05$, vs. AAA before EVAR.
The max diameter of the abdominal aorta in the PPE groups showed a significant increase compared with the control group $(p<0.01)$. According to the definition of AAA, 78.57\% rats in the PPE group (11/14) had formed AAAs (dilation ratio $>150 \%$ ) (Fig. $2 \mathrm{~B}$ and $2 \mathrm{C}$ ). In parallel to aortic dilatation, the aortas from rats induced to have AAAs by PPE exhibited damage to the elastic laminar in- tegrity and waviness (Fig. 3A and 3B). Double fluorescence immunostaining for CD34 (red) and CD309 (green) in aortic sections showed that $\mathrm{CD} 34^{+} / \mathrm{CD} 309^{+}$cells (orange) sharply declined in the PPE group but were abundant in the control group (Fig. 3C).

The blood from the inferior vena cava was then collected from rats with AAAs or controls, and BMNCs were 
isolated using density gradient centrifugation. The percent of circulating $\mathrm{CD} 34^{+} / \mathrm{CD} 309^{+}$cells in BMNCs from rats with definitive AAA was significantly decreased compared

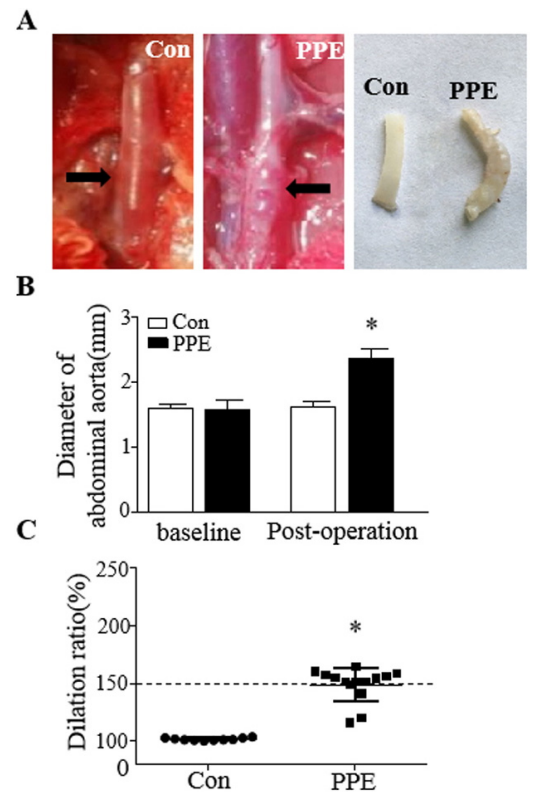

Fig. 2. Aneurysm formation after peri-adventitial elastase application. (A) Characteristic morphology of the abdominal aorta. (Left) One abdominal aortic aneurysm is shown from the Control group. (Middle) An aorta at the end of 5th week after PPE application. (Right) Morphology of a separated abdominal aorta. (B) Changes in the aortic diameter above baseline and post-operatively. (C) The dilation ratios of the abdominal aorta followed for 5 weeks. An aneurysm was defined as an increase in the aortic diameter by $\geq$ $50 \%$ above baseline. ${ }^{*} \mathrm{p}<0.05$, vs. Con. with the control group (Fig. 4).

\section{Discussion}

The present study accessed circulating EPC numbers using specific cell-surface antigens via flow cytometry. The data showed an inverse association between low $\mathrm{CD} 34^{+}$/ $\mathrm{CD} 309^{+}$EPC counts and the presence of AAAs in patients, and this study was the first to demonstrate the consistent and independent association of circulating EPCs in clinical AAAs before or after undergoing EVAR. This study also described a rat model of aneurysm formation by degradation of the elastic lamina. Reduced levels of circulating EPCs occurred in AAA models induced by pancreatic elastase exposure, which suggests that this model of aneurysms formed by peri-adventitial application could advantageous for the future study of AAAs.

The integrity and functionality of the endothelium is crucial for maintaining physiological homeostasis of the entire cardiovascular system (15). Pathological changes in the endothelium can occur in response to adverse environmental factors, including pathogens, inflammatory molecules, or metabolic molecules (16-18), and with advancing age, can cause vascular dysfunctions. Circulating EPCs play a critical role in endothelial repair of vascular damage. Endothelial function and the levels of circulating EPCs have been shown to be associated with cardiovascular risk factors $(4,19)$. Moreover, decreased levels of circulating EPCs can independently predict cardiovascular events.

EPCs are a heterogeneous cell population that can be

$\mathbf{A}$

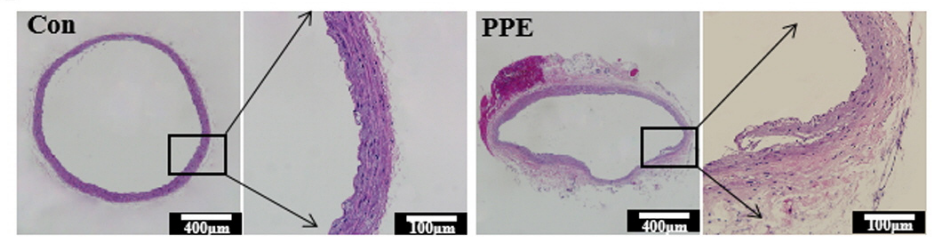

B

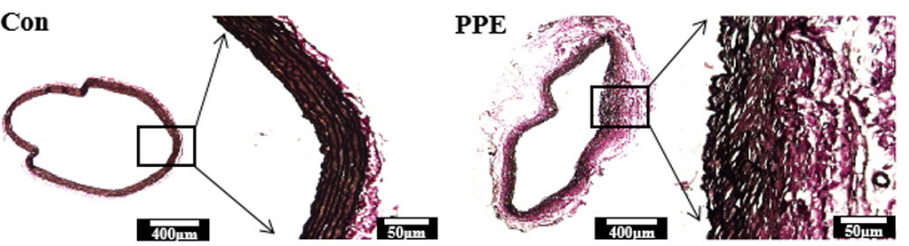

C

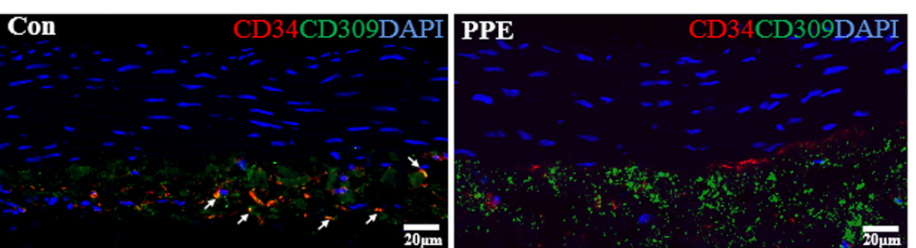

Fig. 3. Histological sections of abdominal aorta. (A) Hematoxylin and eosin staining of the aorta. (B) Verhoeff-Van Gieson (VVG) staining of elastic fibers in aneurysm sections. (C) Immunofluorescence of abdominal aorta sections labeled with antibodies to detect CD34 (red) and CD309 (green). The white arrow indicates $\mathrm{CD} 34^{+} / \mathrm{CD} 309^{+}$cells colocalizing as orange. 
A

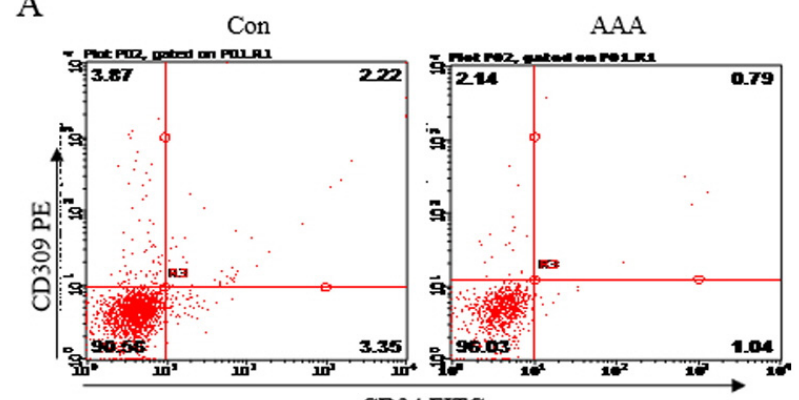

B

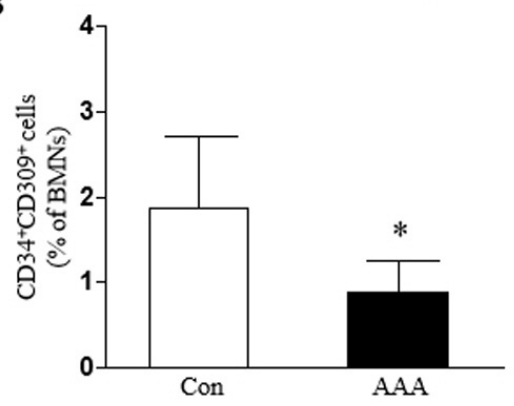

Fig. 4. Circulating levels of EPCs in AAA rats. (A) Circulating numbers of EPCs in different groups by flow cytometry. (B) Percentage of circulating $\mathrm{CD} 34^{+} / \mathrm{CD} 39^{+}$cells in rats with abdominal aortic aneurysms compared with controls. ${ }^{*} p<0.05$, vs. Con.

mobilized from the bone marrow into the peripheral circulation (20). Evidence suggests that EPCs can be characterized by the coexpression of surface markers such as CD34, CD133, and CD309 (21), and can be quantified by flow cytometry in human whole blood or bone marrow (22, 23). CD34 and CD133 are typically considered markers of immaturity and are mainly expressed in hematopoietic stem cells, but CD133 is lost during maturation (24). CD309 often indicates early endothelial differentiation. Therefore, with the progressive loss of $\mathrm{CD} 133, \mathrm{CD} 34^{+} /$ $\mathrm{CD}_{309^{+}}$cells become the main constituents of the circulating EPC pool in the peripheral blood. In this study, we used flow cytometry to analyze the co-expression of CD34 and CD309 as a proxy of the levels of circulating EPCs.

Numerous studies have demonstrated the significant association of altered numbers and impairment of the function of EPCs, with the development of aortic aneurysms (4) and other cardiovascular diseases (25-28). Our findings confirm and suggest that the AAAs in elderly patients with a low level of $\mathrm{CD} 34^{+} / \mathrm{CD} 309^{+} \mathrm{EPCs}$ are larger in aortic diameter and consequently present greater risks of acute complications. Although the exact mechanisms by which EPCs in AAAs improve vascular repair are currently unknown, our data suggests that increased number of EPCs may be important in this scenario.

Previous studies have demonstrated that circulating
EPC levels are reduced and the functions of EPCs are impaired in abdominal aortic aneurysm patients $(7,29)$. Abdominal aortic aneurysm patients who have undergone open AAA repair had significantly higher levels of circulating EPCs in earlier studies $(29,30)$. Low levels of CD34 ${ }^{+}$/ $\mathrm{CD} 39^{+}$in circulating progenitor cells were shown to be associated with increased mortality and risk of incident peripheral arterial disease-related events and myocardial infarction (31). Conversely, both old AAA cases and old hypertensive subjects with aorta dilation showed significantly increased levels of EPC populations for compensative responses (32). Patients with ascending aortic aneurysms had significantly higher levels of circulating EPCs $(6,32)$. Both tricuspid and bicuspid aortic valve patients with thoracic aortic aneurysms displayed a significant elevation in the number of EPCs compared with subjects without thoracic aortic aneurysms (33). These reports contrast with some prior studies, including our present findings (6). Although an opposite quantitative relationship for circulating EPCs was observed, both thoracic aortic and abdominal aortic aneurysm patients show consistent functional impairments of circulating EPCs. Therefore, these differences suggest that EPCs may participate in vascular repair via multiple mechanisms, depending on the severity of arterial dilatation and the pathologic features of tissues (32).

EVAR for abdominal aortic aneurysms is the standard treatment for suitable patients and is associated with reduced perioperative morbidity and cost-effectiveness compared with open surgical repair (34). To our knowledge, this is the first study directly comparing the number of circulating EPCs from AAA patients before or after undergoing EVAR.

Elastase plays a major role in the destruction of elastin within the aortic wall and involves in pathobiology of aortic aneurysms (35). An AAA rat model via intra-arterial infusion of elastase is one of the most popular among several small animal models and was first introduced by Anidjar in 1990 (36). This model can mimic most characteristics of human AAAs, but is still limited by an unacceptably high mortality $(14,37)$. Our present rat model established using the peri-adventitial application of elastase had a $100 \%$ total survival rate, and $78.57 \%$ rats formed AAAs. Moreover, the numbers of circulating $\mathrm{CD} 34^{+} / \mathrm{CD} 309^{+}$ cells in BMNCs from defined AAA rats decreased, consistent with the situation in AAA patients before EVAR. Thus, this aneurysm model established by peri-adventitial application might be better suited to study the role of EPCs in the formation and development of AAAs.

In conclusion, remarkably, in our experience, circulat- 
ing EPCs were significantly inversely correlated with aortic dilation. EPCs are mobilized in AAAs patients after EVAR, perhaps as an ongoing attempt at vascular repair. These findings may explain the potential role of circulating EPCs in the development and progression of AAAs and support the future treatment of EPCs for vascular repair in AAAs.

\section{Acknowledgments}

This study was financially supported from National Natural Science Foundation of China (81700361, 91839101, 81970397) and Introduction Project of Clinical Medicine Expert Team for Suzhou (No. SZYJTD201704). We thank LetPub (www.letpub.com) for its linguistic assistance during the preparation of this manuscript.

\section{Potential Conflict of Interest}

The authors have no conflicting financial interest.

\section{Author Contributions}

Weihua $\mathrm{Wu}$ researched and analysed data, drafted the manuscript. Jinlong Zhang and Lianbo Shao researched and analysed data. Haoyue Huang researched and collected data, and approved the submitted version. Zhenya Shen contributed to the experimental design and discussion, reviewed/edited the manuscript and approved the submitted version. Qingyou Meng revised the manuscript and approved the final version. Xiaomei Teng designed the experiments, researched data, drafted the manuscript and approved the submitted version.

\section{References}

1. Aggarwal S, Qamar A, Sharma V, Sharma A. Abdominal aortic aneurysm: a comprehensive review. Exp Clin Cardiol 2011;16:11-15

2. Sakalihasan N, Limet R, Defawe OD. Abdominal aortic aneurysm. Lancet 2005;365:1577-1589

3. Altobelli E, Rapacchietta L, Profeta VF, Fagnano R. Risk factors for abdominal aortic aneurysm in population-based studies: a systematic review and meta-analysis. Int J Environ Res Public Health 2018;15:2805

4. Parietti E, Pallandre JR, Deschaseaux F, Aupècle B, Durst C, Kantelip JP, Chocron S, Davani S. Presence of circulating endothelial progenitor cells and levels of stromal-derived factor- $1 \alpha$ are associated with ascending aorta aneurysm size. Eur J Cardiothorac Surg 2011;40:e6-e12

5. Hayek SS, MacNamara J, Tahhan AS, Awad M, Yadalam A, Ko YA, Healy S, Hesaroieh I, Ahmed H, Gray B, Sher SS, Ghasemzadeh N, Patel R, Kim J, Waller EK, Quyyumi AA. Circulating progenitor cells identify peripheral arterial disease in patients with coronary artery disease. Circ Res 2016;119:564-571

6. Dawson J, Tooze J, Cockerill G, Choke E, Loftus I, Thompson MM. Endothelial progenitor cells and abdominal aortic aneurysms. Ann N Y Acad Sci 2006;1085:327-330

7. Sung SH, Wu TC, Chen JS, Chen YH, Huang PH, Lin SJ, Shih CC, Chen JW. Reduced number and impaired function of circulating endothelial progenitor cells in patients with abdominal aortic aneurysm. Int J Cardiol 2013;168: 1070-1077

8. Chong MS, Ng WK, Chan JK. Concise review: endothelial progenitor cells in regenerative medicine: applications and challenges. Stem Cells Transl Med 2016;5:530-538

9. Leake AE, Segal MA, Chaer RA, Eslami MH, Al-Khoury G, Makaroun MS, Avgerinos ED. Meta-analysis of open and endovascular repair of popliteal artery aneurysms. J Vasc Surg 2017;65:246-256.e2

10. Ammar AD. Mortality for open abdominal aortic aneurysm repair before and after endovascular aortic repair (EVAR). Am Surg 2019;85:1341-1344

11. Dua A, Koprowski S, Upchurch G, Lee CJ, Desai SS. Progressive shortfall in open aneurysm experience for vascular surgery trainees with the impact of fenestrated and branched endovascular technology. J Vasc Surg 2017;65: 257-261

12. Zhou B, Cao XC, Fang ZH, Zheng CL, Han ZB, Ren H, Poon MC, Han ZC. Prevention of diabetic microangiopathy by prophylactic transplant of mobilized peripheral blood mononuclear cells. Acta Pharmacol Sin 2007;28:89-97

13. Zheng H, Shen CJ, Qiu FY, Zhao YB, Fu GS. Stromal cell-derived factor lalpha reduces senescence of endothelial progenitor subpopulation in lectin-binding and DiLDL-uptaking cell through telomerase activation and telomere elongation. J Cell Physiol 2010;223:757-763

14. Hu G, Dong Z, Fu W. A novel modification of the murine elastase infusion model of abdominal aortic aneurysm formation. Ann Vasc Surg 2017;42:246-253

15. Olivieri F, Pompilio G, Balistreri CR. Endothelial progenitor cells in ageing. Mech Ageing Dev 2016;159:1-3

16. Weng $\mathrm{X}, \mathrm{Yu} \mathrm{L}$, Liang $\mathrm{P}$, Chen $\mathrm{D}$, Cheng $\mathrm{X}$, Yang $\mathrm{Y}, \mathrm{Li}$ L, Zhang T, Zhou B, Wu X, Xu H, Fang M, Gao Y, Chen $\mathrm{Q}, \mathrm{Xu}$ Y. Endothelial MRTF-A mediates angiotensin II induced cardiac hypertrophy. J Mol Cell Cardiol 2015;80:2333

17. Balistreri CR, Ruvolo G, Lio D, Madonna R. Toll-like receptor-4 signaling pathway in aorta aging and diseases: "its double nature". J Mol Cell Cardiol 2017;110:38-53

18. Zhang H, Liu J, Qu D, Wang L, Wong CM, Lau CW, Huang Y, Wang YF, Huang H, Xia Y, Xiang L, Cai Z, Liu P, Wei Y, Yao X, Ma RCW, Huang Y. Serum exosomes mediate delivery of arginase 1 as a novel mechanism for endothelial dysfunction in diabetes. Proc Natl Acad Sci U S A 2018;115:E6927-E6936

19. Huang PH, Chen YH, Chen YL, Wu TC, Chen JW, Lin SJ. Vascular endothelial function and circulating endothelial progenitor cells in patients with cardiac syndrome $\mathrm{X}$. 
Heart 2007;93:1064-1070

20. Li DW, Liu ZQ, Wei J, Liu Y, Hu LS. Contribution of endothelial progenitor cells to neovascularization (Review). Int J Mol Med 2012;30:1000-1006

21. Schatteman G. Are circulating CD133 + cells biomarkers of vascular disease? Arterioscler Thromb Vasc Biol 2005;25: 270-271

22. Etemadifar M, Dehghani L, Ganji H, Soleimani R, Talebi M, Eskandari N, Samani FS, Meamar R. Evaluation of the circulating CD34(+), CD309(+), and endothelial progenitor cells in patients with first attack of optic neuritis. Adv Biomed Res 2015;4:151

23. Fritzenwanger M, Lorenz F, Jung C, Fabris $M$, Thude H, Barz D, Figulla HR. Differential number of CD34+, CD133+ and CD34+/CD133+ cells in peripheral blood of patients with congestive heart failure. Eur J Med Res 2009;14:113-117

24. Friedrich EB, Walenta K, Scharlau J, Nickenig G, Werner N. CD34-/CD133+/VEGFR-2+ endothelial progenitor cell subpopulation with potent vasoregenerative capacities. Circ Res 2006;98:e20-e25

25. Liao YF, Chen LL, Zeng TS, Li YM, Fan Yu, Hu LJ, Ling Yue. Number of circulating endothelial progenitor cells as a marker of vascular endothelial function for type 2 diabetes. Vasc Med 2010;15:279-285

26. Gu S, Zhang W, Chen J, Ma R, Xiao X, Ma X, Yao Z, Chen Y. EPC-derived microvesicles protect cardiomyocytes from Ang II-induced hypertrophy and apoptosis. PLoS One 2014; 9:e85396

27. Huang WP, Yin WH, Chen JS, Huang PH, Chen JW, Lin SJ. Fenofibrate reverses dysfunction of EPCs caused by chronic heart failure. J Cardiovasc Transl Res 2020;13:158170

28. Fadini GP, Coracina A, Baesso I, Agostini C, Tiengo A, Avogaro A, de Kreutzenberg SV. Peripheral blood CD34+ $\mathrm{KDR}+$ endothelial progenitor cells are determinants of subclinical atherosclerosis in a middle-aged general population. Stroke 2006;37:2277-2282

29. Eizawa T, Ikeda U, Murakami Y, Matsui K, Yoshioka T, Suzuki C, Takahashi M, Muroi K, Kamisawa O, Fuse K,
Shimada K. Increase in circulating endothelial progenitor cells after aortic aneurysm repair. Heart Vessels 2004;19: $107-110$

30. Lacquaniti A, Giardina M, Lucisano S, Messina R, Buemi A, Risitano CD, Chirico V, Buemi M, David A. Neutrophil gelatinase-associated lipocalin (NGAL) and endothelial progenitor cells (EPCs) evaluation in aortic aneurysm repair. Curr Vasc Pharmacol 2013;11:1001-1010

31. Rigato M, Avogaro A, Fadini GP. Levels of circulating progenitor cells, cardiovascular outcomes and death: a metaanalysis of prospective observational studies. Circ Res 2016; 118:1930-1939

32. Buffa S, Borzì D, Chiarelli R, Crapanzano F, Lena AM, Nania M, Candi E, Triolo F, Ruvolo G, Melino G, Balistreri CR. Biomarkers for vascular ageing in aorta tissues and blood samples. Exp Gerontol 2019;128:110741

33. Balistreri CR, Crapanzano F, Schirone L, Allegra A, Pisano C, Ruvolo G, Forte M, Greco E, Cavarretta E, Marullo AGM, Sciarretta S, Frati G. Deregulation of Notch1 pathway and circulating endothelial progenitor cell (EPC) number in patients with bicuspid aortic valve with and without ascending aorta aneurysm. Sci Rep 2018;8:13834

34. Canning P, Tawfick W, Whelan N, Hynes N, Sultan S. Cost-effectiveness analysis of endovascular versus open repair of abdominal aortic aneurysm in a high-volume center. J Vasc Surg 2019;70:485-496

35. Bhamidipati CM, Mehta GS, Lu G, Moehle CW, Barbery C, DiMusto PD, Laser A, Kron IL, Upchurch GR Jr, Ailawadi G. Development of a novel murine model of aortic aneurysms using peri-adventitial elastase. Surgery 2012; 152:238-246

36. Anidjar S, Salzmann JL, Gentric D, Lagneau P, Camilleri JP, Michel JB. Elastase-induced experimental aneurysms in rats. Circulation 1990;82:973-981

37. Busch A, Chernogubova E, Jin H, Meurer F, Eckstein HH, Kim M, Maegdefessel L. Four surgical modifications to the classic elastase perfusion aneurysm model enable haemodynamic alterations and extended elastase perfusion. Eur J Vasc Endovasc Surg 2018;56:102-109 